\title{
Guías «primera línea» para la atención de pacientes con COVID-19. Posición prona en pacientes despiertos no intubados con SDRA
}

«First line guidelines» for the care of patients with COVID-19. Prone position in awake non-intubated patients with ARDS Diretrizes de «primeira linha» para o cuidado de pacientes com COVID-19.

Posição prona em pacientes acordados não intubados com SDRA

Ernesto Deloya-Tomas, ${ }^{*}$ Orlando Rubén Pérez-Nieto, ${ }^{*}$ Eder Iván Zamarrón-López, ${ }^{\ddagger}$

Raúl Soriano-Orozco, ${ }^{\S}$ Cristóbal Meneses-Olguín," Manuel A Guerrero-Gutiérrez,"

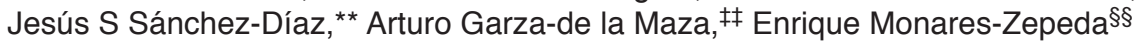

\section{RESUMEN}

Se ha propuesto el uso de posición prona (PP) para pacientes despiertos, no intubados, hospitalizados por neumonía e insuficiencia respiratoria. Esta terapia no es nueva, puesto que se ha descrito durante dos décadas. Se han realizado estudios clínicos observacionales y algunos prospectivos con un número limitado de pacientes y se ha observado una mejora significativa en el aumento de la presión parcial arterial de oxígeno $\left(\mathrm{PaO}_{2}\right)$, disminución de la frecuencia respiratoria y disminución de la insuficiencia respiratoria. La utilidad de la PP en pacientes despiertos aún es controvertida cuando se intenta demostrar que podría prevenir significativamente la intubación.

Palabras clave: Posición prono, paciente no intubado, falla respiratoria, COVID-19, guías.

\section{ABSTRACT}

The use of the prone position (PP) has been proposed for awake, nonintubated patients hospitalized for pneumonia and respiratory failure. This therapy is not new, it has been described for two decades. However, observational and some prospective clinical studies have been conducted with a limited number of patients, and a significant improvement in increased arterial partial pressure of oxygen $\left(\mathrm{paO}_{2}\right)$, decreased respiratory rate, and decreased respiratory failure has been observed. .The usefulness of PP in awake patients remains controversial when trying to show that it could significantly prevent intubation.

Keywords: Prone position, non-intubated patient, respiratory failure, COVID-19, guidelines.

\section{RESUMO}

O uso da posição prona (PP) tem sido proposto para pacientes acordados, não intubados, hospitalizados por pneumonia e insuficiência respiratória. Esta terapia não é nova, é descrita há duas décadas. Estudos observacionais e alguns estudos clínicos prospectivos foram realizados com um número limitado de pacientes e uma melhora significativa foi observada no aumento da pressão parcial arterial de oxigênio $\left(\mathrm{PaO}_{2}\right)$, diminuição da frequência respiratória e diminuição da insuficiência respiratória. A utilidade da PP em pacientes acordados permanece controversa quando se tenta mostrar que ela pode prevenir significativamente a intubação.

Palavras-chave: Posição prona, paciente não intubado, insuficiência respiratória, COVID-19, guias.

\footnotetext{
* Hospital General San Juan del Río, Querétaro.

‡ Hospital CEMAIN Tampico, Tamaulipas, México.

$\S$ Hospital de Alta Especialidad T1 León, Guanajuato, México.

『 Hospital Santo Tomás, Querétaro, Querétaro.

" Instituto Nacional de Cancerología, Ciudad de México, México.

" Unidad de Alta Especialidad «Adolfo Ruíz Cortines», Veracruz, México.

¥¥ Instituto Nacional de Cardiología «Dr. Ignacio Chávez», Ciudad de México, México.

$\$ \S$ Hospital General de México, Ciudad de México, México.

Recibido: 01/02/2021. Aceptado: 19/02/2021.
}

Citar como: Deloya-Tomas E, Pérez-Nieto OR, Zamarrón-López El, Soriano-Orozco R, Meneses-Olguín C, Guerrero-Gutiérrez MA et al. Guías «primera línea» para la atención de pacientes con COVID-19. Posición prona en pacientes despiertos no intubados con SDRA. Med Crit. 2021;35(3):157-161. https://dx.doi.org/10.35366/100006

www.medigraphic.com/medicinacritica

\section{RECOMENDACIONES}

1. Recomendamos los siguientes criterios como contraindicaciones absolutas para la PP despierto: pacientes que requieren intubación inmediata a consideración de un experto, compromiso de las vías respiratorias, fatiga respiratoria, falta de cooperación del paciente, deterioro neurológico, inestabilidad hemodinámica, inestabilidad espinal, inestabilidad pélvica, traumatismo torácico, no contar con el personal y nivel de monitoreo suficiente para la monitorización y cuidado del paciente.

- Semáforo: verde

2. Recomendamos que todo paciente despierto con insuficiencia respiratoria: $\mathrm{SpO}_{2}<92 \%$ y FR $>28$ respiraciones por minuto, sin contraindicaciones absolutas para realizar la maniobra de posición prona (PP), se coloque en dicha posición y se evalúe la respuesta a esta maniobra mediante el índice de ROX y la escala de trabajo respiratorio como mínimo de manera horaria.

\section{- Semáforo: amarillo}

3. Recomendamos que todos los pacientes en PP despierto deben ser monitorizados mediante $\mathrm{SpO}_{2}$ y frecuencia respiratoria de manera continua. Una FR > 28 respiraciones por minuto sostenidas durante más de 30 minutos debe alertar al equipo de respuesta rápida y solicitar evaluación por un experto. - Semáforo: verde

4. Recomendamos el empleo del índice de ROX y la escala de trabajo respiratorio para la monitorización horaria del paciente. Pacientes con índice de ROX $>4.88$ pueden permanecer en vigilancia estrecha en PP despierto sin requerir de intubación. Cifras < 4.88 podrían ameritar intubación en las primeras 12 horas.

- Semáforo: amarillo 
5. Recomendamos que la tolerancia por parte del paciente a la PP despierto $<2$ horas por episodio 0 menos de tres episodios al día debe ser considerada como un fracaso de esta maniobra.

— Semáforo: amarillo

6. Recomendamos definir como éxito de la maniobra que el paciente pueda mantener episodios $>2$ horas al día, manteniendo un índice de ROX sostenido $>$ 4.8 o trabajo respiratorio $\leq 4$ puntos.

- Semáforo: amarillo

7. Recomendamos que cuando el paciente se encuentra en decúbito supino y presente una $\mathrm{SaO}_{2}>92 \%$, con $\mathrm{O}_{2}$ suplementario $<4 \mathrm{~L} / \mathrm{min}$, frecuencia respiratoria $<26$ respiraciones por minuto y una escala de disnea $<2$ /actividad $<3$ modificada (Tabla 1), la PP puede ser descontinuada. ${ }^{1}$

- Semáforo: amarillo

\section{INTRODUCCIÓN}

Durante la pandemia generada por el virus SARSCoV-2, que provoca la enfermedad por coronavirus-2019 (COVID-19), se ha propuesto el uso de posición prona (PP) para pacientes despiertos, no intubados, hospitalizados por neumonía e insuficiencia respiratoria, pero esta terapia no es nueva, se ha descrito durante dos décadas; ${ }^{2}$ sin embargo, su uso se popularizó en todo el mundo durante el último año debido a la gran cantidad de pacientes con insuficiencia respiratoria aguda con el deseo de mejorar la oxi- genación y tratar de evitar la necesidad de intubación y ventilación mecánica invasiva (VMI) debido a la falta de ventiladores mecánicos y camas libres en las Unidades de Cuidados Intensivos (UCI) en muchos hospitales. No obstante, se han realizado estudios clínicos observacionales y algunos prospectivos con un número limitado de pacientes y se ha observado una mejora significativa en el aumento de la presión parcial arterial de oxígeno $\left(\mathrm{PaO}_{2}\right)$, disminución de la frecuencia respiratoria y de la insuficiencia respiratoria. La utilidad de la PP en pacientes despiertos aún es controvertida cuando se intenta demostrar que podría prevenir significativamente la intubación.

\section{CONSIDERACIONES FISIOPATOLÓGICAS PARA EL SDRA Y LA POSICIÓN PRONA}

El síndrome de distrés respiratorio agudo (SDRA) se caracteriza por una inflamación progresiva de la unidad alvéolo-capilar con infiltración de células inflamatorias y formación de membranas hialinas que afecta significativamente el intercambio de gases. Puede desencadenarse por una lesión pulmonar directa, como neumonías víricas (por ejemplo: SARS-CoV-2, virus de la influenza, etcétera) o por enfermedades sistémicas graves (por ejemplo: sepsis, quemaduras, traumatismos múltiples, etcétera). La respuesta inflamatoria local y/o sistémica genera una lesión endotelial a nivel de los capilares pulmonares, aumento de la permeabilidad a los fluidos de la membrana alvéolo-capilar, edema intersticial, edema alveolar, diapédesis y activación de linfocitos, neutrófilos y macrófagos a nivel pulmonar, que generan una

Tabla 1: Escala de disnea-actividad para pacientes hospitalizados.

\begin{tabular}{cccc}
\hline Grado & Nivel de actividad & Grado & Grado de disnea \\
\hline 0 & Puede deambular sin ayuda & 0 & Ausente \\
1 & Puede deambular con ayuda & 1 & Leve \\
2 & Puede estar sentado fuera de cama & 2 & Moderada \\
3 & Puede sentarse en cama & 3 & Grave \\
4 & Todo el tiempo en cama & 4 & Muy grave \\
\hline
\end{tabular}

Se otorga un punto al grado de disnea y se registra el grado de actividad en el que se provocó disnea.

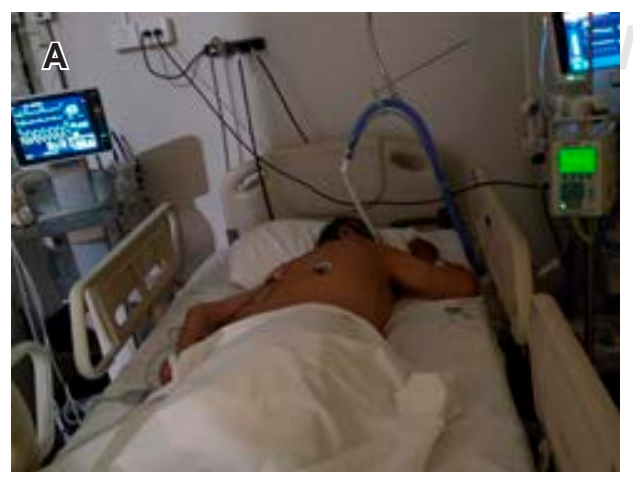

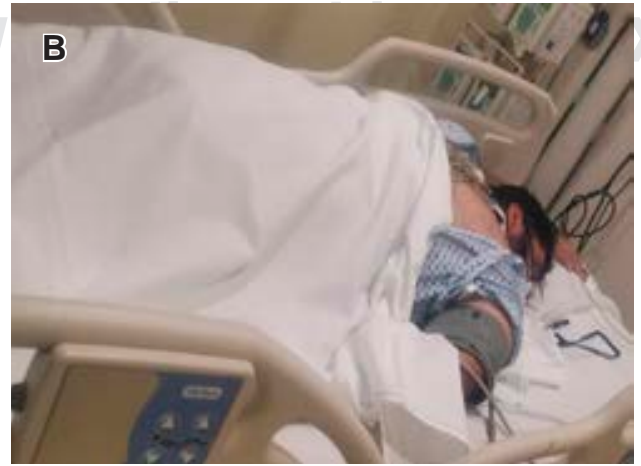

Figura 1:

A y B) Ejemplos de posición prona en pacientes despiertos. Fotografía del archivo personal de los autores con permiso de los pacientes. 
Tabla 2: Pasos para realizar posición prona despierto.

\begin{tabular}{|c|c|}
\hline \multicolumn{2}{|r|}{ Lista de cotejo preprona } \\
\hline Indicaciones & Contraindicaciones \\
\hline $\begin{array}{l}\text { Falla respiratoria } \\
\text { aguda hipoxémica }\end{array}$ & $\begin{array}{l}\text { SDRA con incremento del trabajo respiratorio } \\
\text { Índice de } \mathrm{ROX}<4.8\end{array}$ \\
\hline Paciente & $\begin{array}{l}\text { Trabajo respiratorio }>4 \text {, índice de disnea } \geq 2 \text { actividad }>2 \\
\text { Inestabilidad hemodinámica o arritmias }\end{array}$ \\
\hline consciente y alerta & $\begin{array}{l}\mathrm{PaO}_{2} / \mathrm{FiO}_{2} \text { menor de } 100 \text { con uso de VMNi o CNAF } \\
\text { Estado mental alterado: agitación, convulsiones } \\
\text { Inestabilidad de la caja torácica o espinal } \\
\text { Cirugía abdominal mayor reciente }\end{array}$ \\
\hline
\end{tabular}

SDRA = síndrome de distrés respiratorio agudo, $\mathrm{VMNi}$ = ventilación mecánica no invasiva, CNAF = cánulas nasales de alto flujo.

mayor cantidad de citocinas inflamatorias que incluyen metabolitos derivados del ácido araquidónico e interleucinas, activación del complemento, especies reactivas de oxígeno y proteasas, así como un aumento en la acción de factores protrombóticos que pueden generar trombosis de los capilares pulmonares.

Los mecanismos de hipoxemia implicados en el SDRA son múltiples: disminución de la difusión de oxígeno, hipoventilación, incremento en los cortocircuitos y alteración de la ventilación/perfusión (V/Q). Clínicamente el SDRA se manifiesta por disnea, taquipnea y aumento de la carga en los músculos respiratorios; los estudios de gases arteriales muestran una disminución de la $\mathrm{PaO}_{2}$, una disminución de la saturación parcial de oxígeno arterial $\left(\mathrm{SpO}_{2}\right)$ y un aumento de la presión parcial de dióxido de carbono $\left(\mathrm{PaCO}_{2}\right)$. En mecánica ventilatoria hay una disminución importante de la distensibilidad pulmonar estática (Cst).

Existen grandes áreas de colapso alveolar que predominan en las regiones pulmonares dependientes de la gravedad (posterior y basal) y que son más ricas en capilares, por lo que los cambios en la posición corporal generan modificaciones en la mecánica ventilatoria y el intercambio gaseoso. La posición supina no beneficia la mecánica ventilatoria debido al peso del corazón, la pared anterior del tórax y el abdomen que recae sobre las áreas pulmonares dependientes de la gravedad, disminuyendo la Cst y la capacidad residual funcional (CRF), lo que predispone a la formación de atelectasias y shunts que contribuyen a la hipoxemia y la hipoventilación alveolar. ${ }^{3}$ Por otro lado, al colocar a un paciente en posición prona, el peso del corazón, la pared anterior de la caja torácica y el abdomen cae sobre la cama, lo cual libera la presión ejercida sobre los alvéolos dependientes y provoca una rápida mejora de la ventilación. En la mayoría de los casos, debido al aumento de la CRF y del volumen corriente - esto por incremento en la presión transpulmonar-, también se observa una disminución de los cortocircuitos y áreas de atelectasias.

La mejora de la mecánica ventilatoria no es la única ventaja observada en PP en pacientes no intubados.
En el SDRA hay un desreclutamiento capilar debido a microtrombosis y colapso de la vasculatura pulmonar debido a la redistribución mediada por hipoxemia que disminuye la perfusión. Esto ocurre, de manera predominante, en áreas con poca ventilación, lo cual genera un fuerte impacto en el intercambio de gases. La PP favorece una ventilación más homogénea, lo que mejora la oxigenación y favorece el reclutamiento capilar, beneficiando significativamente a la V/Q.

\section{EVIDENCIA DE DECÚBITO PRONO EN PACIENTES NO INTUBADOS CON SDRA}

Se ha demostrado que la PP reduce la mortalidad en pacientes con SDRA bajo VMI; ${ }^{4}$ sin embargo, todavía no hay pruebas sólidas de que esto ocurra en pacientes no intubados. Existen algunos estudios realizados en las últimas dos décadas que demuestran mejoría en la oxigenación en pacientes con insuficiencia respiratoria hipoxémica cuando se coloca en PP; no obstante, la investigación sobre este tema creció exponencialmente el año pasado durante la pandemia de COVID-19.

La posición prona en pacientes despiertos, no intubados con insuficiencia respiratoria aguda hipoxémica resulta en una mejor oxigenación, esto es demostrado por un aumento de $\mathrm{PaO}_{2}$ y $\mathrm{SpO}_{2}$ y $\mathrm{PaO}_{2}$ /fracción de oxígeno inspirado $\left(\mathrm{PaO}_{2} / \mathrm{FiO}_{2}\right)$, el $\mathrm{pH}$ aumenta debido a una disminución en el nivel de $\mathrm{PaCO}_{2}$, y también se observa una disminución de la frecuencia respiratoria y del trabajo respiratorio. La PP no se asocia con deterioro hemodinámico. ${ }^{5}$ Además, se ha demostrado que la PP combinada con ventilación no invasiva (VNI) o cánula nasal de alto flujo (HFNC) en pacientes con síndrome de distrés respiratorio agudo (SDRA) de moderada a grave es segura y eficaz y puede prevenir la intubación. ${ }^{6,7}$ Una ventaja más de la PP sin intubación es que permite al paciente interactuar con la familia durante la hospitalización, favoreciendo la humanización en la asistencia sanitaria. ${ }^{8}$

Debido a la pandemia de COVID-19 y a la alta demanda mundial de asistencia respiratoria, la PP en pacientes despiertos no intubados se ha vuelto popular en China y el interés clínico ha aumentado rápidamente en

Tabla 3: Pasos a seguir tras cambios de posición (prona-supino, supino-prona).

Durante el cambio de posición a prona

Asistir al paciente: explicar el procedimiento ventajas y beneficios y ayudar al paciente en los cambios de posición

Terapia de oxígeno: garantizar un dispositivo de oxígeno adecuado y bien colocado

Monitoreo de oximetría de pulso continua

Ayudar al paciente en los cambios de posición

La posición en Trendelenburg inversa puede ser más confortable

Monitorizar la frecuencia respiratoria y saturación de oxígeno continuas 
Tabla 4: Niveles y tiempos de monitoreo durante posición prona y cambios de posición.

\begin{tabular}{ll}
\hline \multirow{2}{*}{ A los 15 minutos } & Mescartar intolerancia a la posición y desaturación \\
\hline & Mediciones seriadas de $i \mathrm{ROX}\left(\mathrm{SaO}_{2} / \mathrm{FiO}_{2} / \mathrm{FR}\right)$ \\
Si hay desaturación & $\begin{array}{l}\text { Verificar desconexión del dispositivo, si persiste incrementar el aporte de oxígeno o escalar el tipo de soporte respi- } \\
\text { ratorio (VMNi, CNAF) }\end{array}$ \\
Si el paciente tiene intolerancia & Cambiar a posición supino \\
Si el paciente tiene adecuada tolerancia y & Aconsejar permanecer en PP dos a cuatro horas o el mayor tiempo posible que tolere más de 3 episodios al día \\
saturación 92\% & \\
Monitorizar desaturación cada 15 minutos de los cambios de posición
\end{tabular}

$\mathrm{VMNi}$ = ventilación mecánica no invasiva, CNAF = cánulas nasales de alto flujo.

América y Europa. Se ha informado que una estrategia temprana que combina la PP junto con la ventilación no invasiva (VNI) o la cánula nasal de alto flujo (HFNC) se asocia con una menor intubación y mortalidad ${ }^{9}$ y una mejor oxigenación, y puede reducir la tasa de intubación y mortalidad en comparación con pacientes a quienes no se les administró esta terapia. ${ }^{10-12}$ Sin embargo, un estudio de cohorte observacional prospectivo no encontró beneficios en cuanto a la reducción de la tasa de intubación. ${ }^{13}$

\section{CONSIDERACIONES PRÁCTICAS PARA LA POSICIÓN PRONA EN PACIENTES NO INTUBADOS CON INSUFICIENCIA RESPIRATORIA}

Debido a la gran demanda de atención médica por COVID-19, es conveniente incentivar a todos los pacientes con insuficiencia respiratoria: $\mathrm{SpO}_{2}<92-94 \%$ y $\mathrm{FR}>$ 22-28 respiraciones por minuto, a practicar la posición prona, ya que es una maniobra gratuita y de bajo riesgo que requiere una asistencia mínima para realizarse. La seguridad y eficacia de la PP ha sido demostrada en pacientes no intubados, por lo que se puede realizar de forma segura en todas las áreas de atención, incluyendo urgencias, hospitalización y UCl.

Los pacientes que reciben oxígeno suplementario a través de cánulas nasales, máscara de oxígeno, HFNC y VNI pueden colocarse por ellos mismos en PP de manera segura según su tolerancia y comodidad. Se debe considerar que algunos grupos de pacientes más vulnerables (ancianos, obesos, etcétera) pueden requerir ayuda para posicionarse adecuadamente en el PP. Los pacientes deben ser conscientes del procedimiento, estar despiertos, cooperar, poder comunicarse por sí mismos y movilizarse con una asistencia mínima. La duración recomendada de la PP en pacientes no intubados no está clara, pero se puede establecer de acuerdo con la tolerancia del paciente durante un mínimo de dos horas cada 12 horas o hasta 16 horas al día y se pueden probar posiciones alternas, como lateral y semiprona, si el paciente no puede doblarse completamente ${ }^{14}$ (Figura 1 A y B).
Tabla 5: Criterios para suspender la terapéutica de posición prona.
Cuándo parar la posición prona

En caso de criterios de SDRA grave: $\mathrm{PaO}_{2} / \mathrm{FiO}_{2}<1000 \mathrm{SaFi}<190$

$\mathrm{iROX}<2.8$ a las dos horas $\mathrm{y} / 0<3.4$ a las seis horas, sugieren pobre respuesta y requerimiento inmediato de intubación

En caso de éxito definido como saturación de oxígeno mayor a 92\% por más de dos horas, con oxígeno menor a $4 \mathrm{~L} /$ minuto

Las contraindicaciones propuestas para iniciar la PP en pacientes no intubados incluyen: pacientes que requieren intubación inmediata a consideración de un experto, compromiso de las vías respiratorias, fatiga respiratoria, falta de cooperación del paciente, deterioro neurológico, inestabilidad hemodinámica, inestabilidad espinal, inestabilidad pélvica, traumatismo torácico, cirugía abdominal reciente y embarazo en el tercer trimestre. Algunas de estas contraindicaciones pueden ser relativas; sin embargo, se debe tener en cuenta el riesgo de complicaciones (Tablas 2 a 5).

\section{CONCLUSIÓN}

La PP en pacientes despiertos no intubados con insuficiencia respiratoria es una estrategia ventilatoria basada en la fisiología que mejora la oxigenación y es probable que en algunos casos seleccionados pueda disminuir la necesidad de intubación. ${ }^{15}$ Es una intervención que bien realizada por equipos con adecuado entrenamiento implica un riesgo mínimo, que no requiere de nuevos recursos técnicos o materiales. En la actualidad, es una terapia que se está probando en protocolos de investigación como parte del tratamiento de COVID-19. ${ }^{14,16}$

\section{REFERENCIAS}

1. Apigo M. Development of a work of breathing scale and monitoring need of intubation in COVID-19 pneumonia. Crit Care. 2020;24:477. Available in: https://doi.org/10.1186/s13054020-03176-y.

2. Valter C. Response to the prone position in spontaneously breathing patients with hypoxemic respiratory failure. Acta Anaesthesiol Scand. 2003;47(4):416-418. Available in: https:// doi.org/10.1034/j.1399- 6576.2003.00088.x. 
3. Bower G. Protocol for awake prone positioning in COVID-19 patients: to do it earlier, easier, and longer. Crit Care. 2020;24(1):371. Available in: https://doi.org/10.1186/s13054020-03096-X.

4. Guérin C. Prone positioning acute respiratory distress syndrome patients. Ann Trans/ Med. 2017;5(14):289. Available in: https:// doi:10.21037/atm.2017.06.63.

5. Scaravilli V. Prone positioning improves oxygenation in spontaneously breathing nonintubated patients with hypoxemic acute respiratory failure: a retrospective study. J Crit Care. 2015;30(6):1390-1394. Available in: https://doi.org/10.1016/j.jcrc.2015.07.008.

6. Ding L. Efficacy and safety of early prone positioning combined with HFNC or NIV in moderate to severe ARDS: a multi-center prospective cohort study. Crit Care. 2020;24(1):28. Available in: https://doi.org/10.1186/s13054-020-2738-5.

7. Pérez-Nieto OR. Prone positioning combined with highflow nasal cannula in severe noninfectious ARDS. Crit Care. 2020;24(1):114. Available in: https://doi.org/10.1186/s13054020-2821-y.

8. Slessarev M. Patient self-proning with high-flow nasal cannula improves oxygenation in COVID-19 pneumonia. Can J Anaesth. 2020;67(9):1288-1290. Available in: https://doi.org/10.1007/ s12630-020-01661-0.

9. Sun $\mathrm{Q}$. Lower mortality of COVID-19 by early recognition and intervention: experience from Jiangsu province. Ann Intensive Care. 2020;10(1):33. Available in: https://doi.org/10.1186/ s13613-020-00650-2.

10. Caputo N. Early self-proning in awake, non-intubated patients in the emergency department: a single ED's experience during the COVID-19 pandemic. Academic emergency medicine. Academic Emergency Medicine. 2020;27(5):375-378. Available in: https:// doi.org/10.1111/acem.13994.
11. Jagan N. The POSITIONED study: prone positioning in nonventilated coronavirus disease 2019 patients. A retrospective analysis. Crit Care Explor. 2020;2(10):e0229. Available in: https://doi.org/10.1097/CCE.0000000000000229.

12. Thompson A. Prone positioning in awake, nonintubated patients with COVID-19 hypoxemic respiratory failure. JAMA Intern Med. 2020;e203030. Advance online publication. Available in: https:// doi.org/10.1001/jamainternmed.2020.3030.

13. Ferrando C. Awake prone positioning does not reduce the risk of intubation in COVID-19 treated with high-flow nasal oxygen therapy: a multicenter, adjusted cohort study. Crit Care. 2020;24:597. Available in: https://doi.org/10.1186/s13054-02003314-6.

14. Sodhi K. Awake proning: awake proning: current evidence and practical considerations. Indian J Crit Care Med. 2020;24(12):1236-1241. doi: 10.5005/jp-journals-10071-23684. Available in: doi: https://dx.doi.org/10.5005\%2Fjpjournals-10071-23684.

15. Sryma P. Reinventing the wheel in ARDS: awake proning in COVID-19. Archivos de Bronconeumología. 2020;56(11):747749. Available in: https://doi.org/10.1016/j.arbres.2020.06.002.

16. Perez-Nieto OR, Escarraman-Martinez D, Guerrero-Gutierrez $M A$, et al. Awake prone positioning and oxygen therapy in patients with COVID-19: The APRONOX study. medRxiv. 2021.01.27.21250631; Available in: https://doi.org/10.1101/202 1.01.27.21250631.

Correspondencia:

Ernesto Deloya-Tomas

E-mail: deloyajmr@hotmail.com 